\title{
PSEUDOCIRCLES IN DYNAMICAL SYSTEMS
}

\author{
JUDY A. KENNEDY AND JAMES A. YORKE
}

\begin{abstract}
We construct an example of a $C^{\infty}$ map on a 3-manifold which has an invariant set with an uncountable number of components, each of which is a pseudocircle. Furthermore, any map which is sufficiently close (in the $C^{1}$ metric) to the constructed map has a similar set.
\end{abstract}

\section{INTRODUCTION}

In 1982, Handel [Ha] gave an example of a $C^{\infty}$-diffeomorphism of the plane with an extremely pathological strange attractor. The attractor for his diffeomorphism is a plane-separating continuum known to topologists as a pseudocircle. A continuum is a compact, connected, metric space. A continuum $X$ is indecomposable if it cannot be written as the union of two proper subcontinua. A continuum is hereditarily indecomposable if each of its subcontinua is indecomposable. Pseudocircles are hereditarily indecomposable continua. Since pseudocircles are hereditarily indecomposable, they cannot contain any arcs, that is, continua homeomorphic to the unit interval $[0,1]$.

Several modifications of Handel's example have appeared since 1982. These include results by Herman [He], Moeckel [M], and Pommerenke and Rodin [PR]. As a result of these investigations, and many others, it appears that even for smooth dynamical systems, invariants sets are sometimes quite irregular.

In this paper we construct an example of a dynamical system which we feel is not pathological. Hence our approach and our goals are quite different from those in the papers mentioned. The example we construct is a map $F_{0}$ on a 3manifold. In the example there are two compact, connected sets $R_{1}$ and $R_{2}$ which have interior, and a map $F_{0}$, which has the following properties. The map $F_{0}$ is continuous in a neighborhood $N$ of $R_{1} \cup R_{2}$, and $F_{0}\left(R_{i}\right) \supseteq N$ for $i=1,2$, and $F_{0}\left(\partial R_{i}\right)$ is disjoint from $R_{1} \cup R_{2}$ for $i=1,2$.

Write $\mathbb{B}=\left\{q \mid F_{0}^{n}(q) \in R_{1} \cup R_{2}\right.$ for all $\left.n\right\}$. This set $\mathbb{B}$ has uncountably many components, each of which is nowhere dense. Each component corresponds to a sequence $a=\left\{a_{i}\right\}_{0}^{\infty}$, where each $a_{i} \in\{1,2\}$. For each such sequence there is a unique component of $\mathbb{B}$, denoted $B(a)=\left\{q \mid F_{0}^{n}(q) \in R_{a_{n}}\right.$ for $n=$ $0,1,2, \ldots\}$.

Received by the editors September 9, 1992.

1991 Mathematics Subject Classification. Primary 54F20, 54F50, 58F12.

Key words and phrases. Indecomposable invariant set, pseudocircle, $C^{\infty}$ map, perturbable dynamical system. 0033.

This research was supported in part by NSF grant DMS-9006931 and AFOSR grant F49620-J- 
In our case $R_{1}$ and $R_{2}$ are each the product of a disk and a circle. Each component of $B$ is in some sense circlelike, and uncountably many of these components are pseudocircles. The construction depends only on how the maps are defined on $R_{1} \cup R_{2}$ and does not even depend on the 3-manifold in which we place $R_{1} \cup R_{2}$, but for notational simplicity, we choose a specific 3-manifold and define $F_{0}$ on the entire manifold.

In the construction below there is a discontinuity (outside a compact neighborhood of $R_{1} \cup R_{2}$ ), but this discontinuity can be removed by embedding the compact neighborhood in a different 3-manifold such as $\mathbb{R}^{3}$ or $S^{3}$ (in which $R_{1}$ and $R_{2}$ are contractible). Our construction is perturbable in the sense that for any map which is sufficiently close (in the $C^{1}$ topology) to $F_{0}$ we still have uncountably many components of $\mathbb{B}$ that are pseudocircles.

We construct a map $F_{0}$ from $S^{1} \times \mathbb{R}^{1} \times S^{1}$ to itself, write elements in that space $q=(x, y, z)$, and refer to the $x, y$, and $z$ coordinates of $q$ as well as the first, second, and third coordinates of $q$. The objective is to investigate the dynamical system $q_{n+1}=F_{0}\left(q_{n}\right)$. The map is chosen so that for every starting point $q_{0}$ the trajectory $q_{n}$ has its $y$ coordinate go either to $+\infty$ or to $-\infty$, or remain bounded as $n$ approaches $\infty$, and we refer to initial points $q_{0}$ as either $+\infty$-points, $-\infty$-points, or bounded points. Our results concern what happens for particular initial values of $z$. For $z \in S^{1}$, let $\widetilde{B}_{z}=\{(x, y) \mid(x, y, z)$ is a bounded point $\}$. For uncountably many choices of $z, \widetilde{B}_{z}$ is a pseudocircle in $S^{1} \times \mathbb{R}^{1}$ which separates $S^{1} \times \mathbb{R}^{1}$ into two open sets, each homeomorphic to $S^{1} \times \mathbb{R}^{1}$. For each such $z, \widetilde{B}_{z} \times\{z\}$ is a pseudocircle in the space, and the union of these pseudocircles $\widetilde{B}_{z} \times\{z\}$ forms an invariant set (the invariant set mentioned in the abstract) in the space.

There are both advantages and disadvantages associated with our particular construction. Choose disjoint intervals $I_{1}$ and $I_{2}$ in $S^{1}$ such that (1) $\left[-\frac{1}{128}, \frac{41}{128}\right]=I_{1}$ (so that $I_{1}$ is a fattened up $\left[0, \frac{1}{4}\right]$ ), and (2) $\left[\frac{63}{128}, \frac{100}{128}\right]=I_{2}$ (so that $I_{2}$ is a fattened up $\left.\left[\frac{1}{2}, \frac{3}{4}\right]\right)$. The map $F_{0}$ is chosen so that $F_{1}=$ $F_{0} \mid\left(S^{1} \times \mathbb{R}^{1} \times I_{1}\right)$ is a $C^{\infty}$ map, and the map $F_{2}=F_{0} \mid\left(S^{1} \times \mathbb{R}^{1} \times I_{2}\right)$ is chosen so that both the map and its inverse are $C^{\infty}$ maps. However, as long as the space is $S^{1} \times \mathbb{R}^{1} \times S^{1}, F_{0}$ cannot be chosen so that it is continuous and $F_{1}$ cannot be chosen so that it is one-to-one. In the previously mentioned investigations, the sets of interest are attractors or minimal invariant sets, and the maps are $C^{\infty}$ diffeomorphisms. Thus, with regard to these aspects, our example has disadvantages compared to the previous investigations. We hope to change our example by raising the dimension of our space, so that $F_{0}$ will be a diffeomorphism on a manifold of dimension 7 and all diffeomorphisms close to $F_{0}$ will have the same properties. But that will have to be left for future work.

On the advantage side, we believe our example is (1) natural, in the sense that it could actually model a physical or naturally occurring system; and is (2) perturbable. The constructions of the earlier examples goes something like the following: There is a diffeomorphism $h_{1}$ of $\mathbb{R}^{2}$ that satisfies properties (1) to $(n)$. Having $h_{1}$, it is possible to choose a diffeomorphism $h_{2}$ so that not only are properties (1) to $(n)$ satisfied, but also $h_{2}$ "dovetails" with $h_{1}$. Then $h_{3}$ is chosen to "dovetail" with $h_{1}$ and $h_{2}$ and to satisfy properties (1) to $(n)$. The process continues, and the result is a diffeomorphism $h$ on $\mathbb{R}^{2}$ obtained as a limit of the diffeomorphisms, $h_{1}, h_{2}, \ldots$. Thus, $h$, although nice in 
many respects, is somewhat fragile. Such constructions are not perturbable; i.e., diffeomorphisms close to $h$ would admit much simpler (topologically) invariant sets.

Dynamical systems often exhibit expansion or contraction in one or more directions, as well as shearing and rippling behaviors, and attracting or repelling fixed points. Our map is constructed by combining a finite and explicit set of these behaviors. Specifically, two maps, $T$ (for "two") and $W$ (for "wiggle"), are constructed on $S^{1} \times \mathbb{R}^{1}$, and a map $g$ on $S^{1}$ is constructed. Two disjoint intervals $I_{1}$ and $I_{2}$ are selected and the map $F_{0}$ on the $S^{1} \times \mathbb{R}^{1} \times S^{1}$ is chosen so that $F_{0}(x, y, z)=\left(\mu_{z}(x, y), g(z)\right)$ with $\mu_{z}=T$ if $z \in I_{1}$ and $\mu_{z}=W$ if $z \in I_{2}$. (We do not specify $F_{0}$ outside of $S^{1} \times \mathbb{R}^{1} \times\left(I_{1} \cup I_{2}\right)$.) If $S^{1} \times \mathbb{R}^{1} \times S^{1}$ is then embedded in $\mathbb{R}^{3}$ or $S^{3}$ in a nice, smooth way, it is then possible to extend $F_{0}$ defined on the embedded image of $S^{1} \times \mathbb{R}^{1} \times S^{1}$ in a smooth way. We denote the extended map on $\mathbb{R}^{3}$ or $S^{3}$ by $F_{0}$ also, as we can depend on context to avoid ambiguity. Then there exists an open set $u$ in the $C^{1}$ maps on $\mathbb{R}^{3}$ or $S^{3}$ such that $u$ contains $F_{0}$ and such that if $F \in u$, then $F$ also has an invariant set with an uncountable number of pseudocircle components. In this sense, our dynamical system is perturbable.

\section{BACKGROUND AND NOTATION}

If $(X, d)$ is a metric space, let $H(X)=\{h \mid h$ is a homeomorphism from $X$ onto itself $\} . C^{0}(X)=\{f \mid f$ is a continuous map from $X$ to itself $\}$, and, whenever $X$ is a differentiable manifold, $C^{1}(X)=\{f \mid f$ is a differentiable map from $^{\bullet} X$ to itself $\}$. For $\varepsilon>0, h \in H(X), f \in C^{0}(X)$, and $\hat{f} \in C^{1}(X)$, let

$$
\begin{aligned}
N_{\varepsilon}(h) & =\{k \in H(X) \mid d(k(x), h(x))<\varepsilon \text { for } x \in X\}, \\
N_{\varepsilon}^{0}(f) & =\left\{k \in C^{0}(X) \mid d(k(x), f(x))<\varepsilon \text { for } x \in X\right\}, \\
N_{\varepsilon}^{1}(\hat{f}) & =\left\{k \in C^{1}(X) \mid d(k(x), \hat{f}(x))<\varepsilon,\right. \\
& \text { and }\|D k(x)-D \hat{f}(x)\| z<\varepsilon, \text { for each } x \in X\},
\end{aligned}
$$

where $D k$ and $D \hat{f}$ and the Jacobian matrices and $\|\cdot\|$ is a norm on the space of matrices.

For purposes of the proofs the metric spaces that interest us here are $S^{1}$, $S^{1} \times \mathbb{R}^{1}, \mathbb{R}^{1} \times \mathbb{R}^{1}=\mathbb{R}^{2}, S^{1} \times \mathbb{R}^{1} \times S^{1}$, and $\mathbb{R}^{1} \times \mathbb{R}^{1} \times S^{1}$. Since we consider $S^{1}$ as $\mathbf{R}^{1} \bmod 1$, for $a, b \in S^{1}$, we take the distance from $a$ to $b$ in $S^{1}$ to be $\operatorname{dist}(a, b)$, the length of the shorter arc in $S^{1}$ between $a$ and $b$. For $(x, y)$ and $\left(x^{\prime}, y^{\prime}\right) \in \mathbb{R}^{2}$, the distance from $(x, y)$ to $\left(x^{\prime}, y^{\prime}\right)$ is $\sqrt{\left(x-x^{\prime}\right)^{2}+\left(y-y^{\prime}\right)^{2}}$, and for $(x, y)$ and $\left(x^{\prime}, y^{\prime}\right) \in S^{1} \times \mathbb{R}^{1}$, the distance from $(x, y)$ to $\left(x^{\prime}, y^{\prime}\right)$ is $\sqrt{\left(\operatorname{dist}\left(x, x^{\prime}\right)\right)^{2}+\left(y-y^{\prime}\right)^{2}}$. Distances in $S^{1} \times \mathbb{R}^{1} \times S$ and $\mathbb{R}^{1} \times \mathbb{R}^{1} \times S^{1}$ are defined analogously. In all three cases, $d$ denotes distance, i.e., for $a$ and $b$ points of one of these spaces, $d(a, b)$ denotes the distance from $a$ to $b$ in the space. We rely on the context to avoid ambiguity. Likewise, if $\varepsilon>0$ and $q$ is a point is in one of our spaces, then $D_{\varepsilon}(q)$ denotes the set of all points of the space at a distance less than $\varepsilon$ from $q$.

We use $\pi_{1}, \pi_{2}$ to denote the projections of $S^{1} \times \mathbb{R}^{1}$ or $\mathbb{R}^{1} \times \mathbb{R}^{1}$ to their respective coordinate spaces. When discussing $S^{1} \times \mathbb{R}^{1} \times S^{1}$ we speak of $\pi_{3}$ as well. Let $\mathbb{N}$ denote the positive integers and $\mathscr{Z}$ denote the integers.

A finite collection $C=\left\{c_{0}, c_{1}, \ldots, c_{n}\right\}$ of sets is a circular chain if $c_{i} \cap c_{j} \neq$ 
$\varnothing$ if and only if $|i-j| \leq 1$ or $\{i, j\}=\{0, n\}$. The elements of a circular chain are called links. In this paper the circular chains have links that are closures of open sets, and adjacent links intersect only at their boundaries. A continuum is circularly chainable or circlelike if for each $\varepsilon>0$, it has a circular chain cover of mesh less than $\varepsilon$. Pseudocircles can be characterized as circularly chainable, hereditarily indecomposable continua which can be embedded in any 2-manifold. A pseudocircle embedded in a cylinder $\left(S^{1} \times \mathbb{R}^{1}\right)$ separates that cylinder into two open, connected sets. Pseudocircles have been widely studied, and have many startling properties. For most mathematicians these objects are outside the intuition and seem to be without structure. Quite the reverse is true: these objects have a rich structure. For more information and references regarding pseudocircles we refer the reader to [B, F1, F2, R, KR].

Finally, when proving that a continuum $X$ is hereditarily indecomposable, the following fundamental theorem is often useful. It appeared in the form given here in [OT], but it is due to Krasinkiewicz and $\mathrm{Minc}$ [ $\mathrm{Kr}, \mathrm{KM}]$.

Kransinkiewicz-Minc Theorem. Let $X$ be a continuum. Then the following are equivalent:

(I) $X$ is hereditarily indecomposable.

(II) For every pair of disjoint, closed subsets $A$ and $B$ of $X$ and for every open set $U$ intersecting all components of $A$, there exist closed subsets $M$ and $N$ of $X$ such that

(1) $X=M \cup N$,

(2) $A \subseteq M, B \subseteq N$, and

(3) $M \cap N \subseteq U-A \cup B$.

(III) For every pair of disjoint, closed subsets $A$ and $B$ of $X$ and for every pair of neighborhoods $U$ of $A$ and $V$ of $B$, there exist closed subsets $X_{0}, X_{1}$, and $X_{2}$ of $X$ such that

(1) $X=X_{0} \cup X_{1} \cup X_{2}$,

(2) $A \subseteq X_{0}$ and $B \subseteq X_{2}$,

(3) $X_{0} \cap X_{2}=\varnothing$, and

(4) $X_{0} \cap X_{1} \subseteq V$ and $X_{1} \cap X_{2} \subseteq U$.

\section{RESULTS AND PROOFS}

First, we define maps $\alpha, \sigma, s$, and $T$ on $S^{1} \times \mathbb{R}^{1}$ and $\mathbb{R}^{1} \times \mathbb{R}^{1}$. (We rely on context to tell whether the space involved is $S^{1} \times \mathbb{R}^{1}$ or $\mathbb{R}^{1} \times \mathbb{R}^{1}$. The maps defined make sense in both spaces, the difference being that in $S^{1} \times \mathbb{R}^{1}$, $(x, y)=(x+1, y)$. That is, we think of $S^{1}$ as the quotient space $\mathbb{R}^{1} / \mathscr{Z}$.)

For $(x, y) \in S^{1} \times \mathbb{R}^{1}$ or $\mathbb{R}^{1} \times \mathbb{R}^{1}$, define $\sigma(x, y)=(x-y, y), s(x, y)=$ $(x, 128 y-127 \sin 2 \pi x)=(x, y+127(y-\sin 2 \pi x))$, and $T(x, y)=(2 x, 2 y)$. Note that $\sigma$ and $s$ are homeomorphisms in both spaces with $\sigma^{-1}(x, y)=$ $(x+y, y)$ and $\left.s^{-1}(x, y)=\left(x, \frac{1}{128} y+\frac{127}{128} \sin 2 \pi x\right)\right)$. The map $T$ is a homeomorphism on $\mathbb{R}^{1} \times \mathbb{R}^{1}$ but not on $S^{1} \times \mathbb{R}^{1}$, with $T^{-1}(x, y)=\left(\frac{1}{2} x, \frac{1}{2} y\right)$ for $(x, y) \in \mathbb{R}^{1} \times \mathbb{R}^{1}$. Also, $T: S^{1} \times \mathbb{R}^{1} \rightarrow S^{1} \times \mathbb{R}^{1}$ is not homotopic to the identity. However, the lift $T: \mathbb{R}^{1} \times \mathbb{R}^{1} \rightarrow \mathbb{R}^{1} \times \mathbb{R}^{1}$ is a diffeomorphism homotopic to the identity.

Let $B_{0}=S^{1} \times\{0\}$, or, depending on context to avoid ambiguity, $\mathbb{R}^{1} \times\{0\}$. Likewise, let $A_{0}=S^{1} \times[-2,2]$ or $\mathbb{R}^{1} \times[-2,2]$. Let $R=\{(x, y) \mid y=\sin 2 \pi x\}$. 


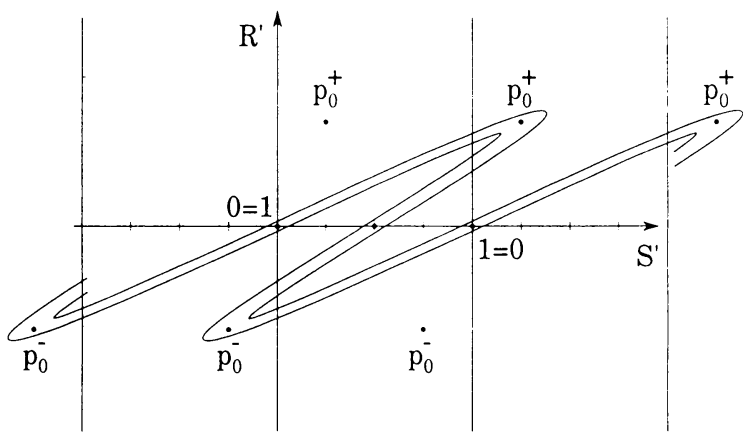

Figure 1

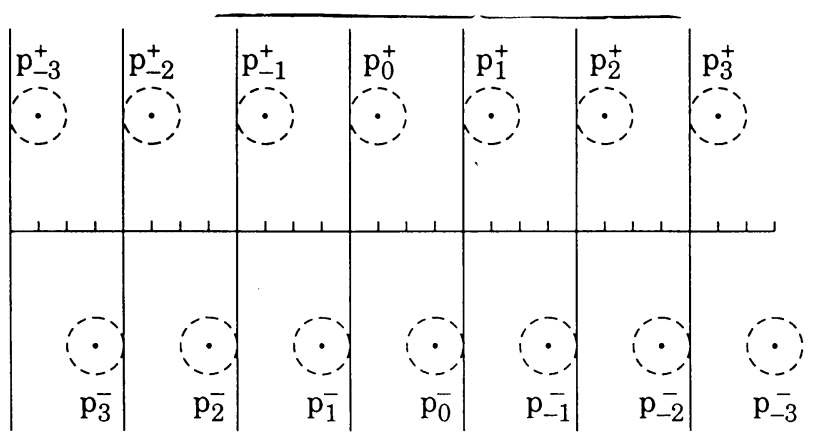

Figure 2

Thus, $R$ is the graph of the sine function with $R \subseteq \mathbb{R}^{1} \times \mathbb{R}^{1}$ and $R \subseteq S^{1} \times \mathbb{R}^{1}$ (depending on context). Note that each point of $R$ is fixed under $s$. See Figure 1.

Let $p_{0}^{+}=\left(\frac{1}{4}, 1\right)$ and $p_{0}^{-}=\left(\frac{3}{4},-1\right)$. For $n \in \mathscr{Z}, p_{n}^{+}=\left(n+\frac{1}{4}, 1\right)$ and $p_{n}^{-}=\left(\frac{3}{4}-n,-1\right)$. (In $S^{1} \times \mathbb{R}^{1}, p_{n}^{+}=p_{0}^{+}$for all $n$ and $p_{n}^{-}=p_{0}^{-}$for all $n$. See Figure 2. Note that $\sigma^{-1}\left(p_{i}^{ \pm}\right)=p_{i+1}^{ \pm}$.)

For $(x, y) \notin D_{1 / 4}\left(p_{n}^{+}\right) \cup D_{1 / 4}\left(p_{n}^{-}\right)$for any $n, \alpha(x, y)=(x, y)$. For $n \in \mathscr{Z}$, $p=p_{n}^{+}$or $p_{n}^{-}$, each point $(x, y)$ in $\overline{D_{1 / 4}(p)}-\{p\}$ is on exactly one radial line $L$ through $p$ in $\overline{D_{1 / 4}(p)}$. Define $\alpha$ so that $\alpha(L)=L$ with $\alpha(p)=p$. Further, if $(x, y) \in \overline{D_{3 / 16}(p)}$, then $d_{(x, y)}=d(p,(x, y)) \leq \frac{3}{16}$. Define $\alpha(x, y)$ to be that unique point on $L$ at a distance of $d_{(x, y)}^{4}+2^{-7} d_{(x, y)}=d_{(x, y)}+\left(d_{(x, y)}^{3}+2^{-7}\right)$. For $(x, y)$ outside $\overline{D_{3 / 16}(p)}$ but in $D_{1 / 4}(p)$, define $\alpha(x, y)$ in such a way that $\alpha(x, y)$ is a point on $L$ closer to $p$ than is $(x, y)$ and in such a way that the resulting $\alpha$ is a diffeomorphism (on both $\mathbb{R}^{1} \times \mathbb{R}^{1}$ and $S^{1} \times \mathbb{R}^{1}$ ). Let $W=\alpha \sigma^{-1} s^{-1}$. Then $W^{-1}=s \sigma \alpha^{-1}$. See Figure 3 .

For a finite collection $f_{1}, f_{2}, \ldots, f_{n}$ of maps on a space, let $\hat{f}_{n}=f_{n} \circ f_{n-1}$ 。 $\cdots \circ f_{1}$.

Lemmas 1-4 and Theorem 5 prove that (1) it is possible to choose a sequence $g_{1}, g_{2}, \ldots$ of the maps $T$ and $W^{-1}$ so that $\bigcap_{n=1}^{\infty}\left(\hat{g}_{n}\right)^{-1}\left(A_{0}\right)$ is a pseudocircle, and (2) for each choice of sequence $g_{1}, g_{2}, \ldots$ of maps $T$ and $W^{-1}$, $\bigcap_{n=1}^{\infty}\left(\hat{g}_{n}\right)^{-1}\left(A_{0}\right)$ is a continuum that separates $S^{1} \times \mathbb{R}^{1}$ into two disjoint, open sets each homeomorphic to $S^{1} \times \mathbb{R}^{1}$. Further, they show that even if the respective sequences consist of maps close to the maps $T$ and $W^{-1}$, then the 


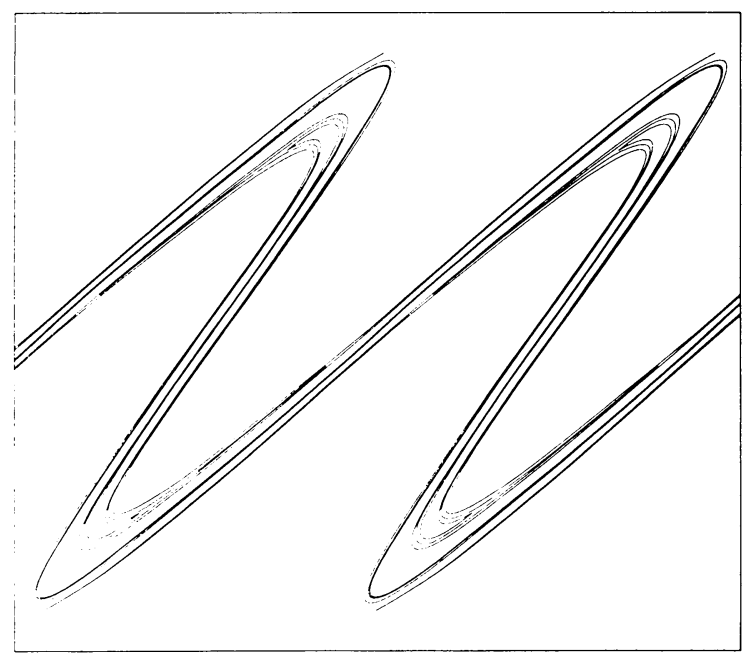

FIgURE 3. Computer generated approximation of $W^{3}\left(A_{0}\right)$

results hold. In order to prove our final result, it is necessary to extend these results to maps on $S^{1} \times \mathbb{R}^{1} \times S^{1}$, but the lemmas, theorem, and proofs given here generalize easily to that situation, which is discussed later.

It may help, as far as understanding the arguments here, to remember that the map $W$ gives the indecomposability present in the invariant set, and that Lemmas 4 and $4^{\prime}$ make this precise. Lemmas 1 and 2 work together to give Lemma 4 and then Lemma $4^{\prime}$. However, the indecomposability given the invariant set by $W$ is only an indecomposability "in the large." To give the hereditary indecomposability it is necessary to adjust the sizes of the "zigzags" associated with $W$ so that all sizes are present, and that is the role of $T$ in the construction. Lemma 3 makes the properties of $T$ precise.

Lemma 1 (Space: $S^{1} \times \mathbb{R}^{1}$ ). Let $\varepsilon$ be a positive number less than $2^{-7}$. Suppose that for each $i \in\{1, \ldots, n\}, W_{i} \in N_{\varepsilon / \pi}(W)$. Then if $p=p_{0}^{+}$or $p_{0}^{-}$, and $q \in D_{1 / 16 \pi+\varepsilon / \pi}(p)$, then $\widehat{W}_{n}(q) \in \overline{D_{1 / 16 \pi+\varepsilon / \pi}(p)}$.

Proof. For $\beta<\frac{1}{4},(1-\cos (2 \pi \beta))^{2}+(\sin (2 \pi \beta))^{2}<2 \pi \beta$. (Consider the unit circle.) This leads to the inequality

$$
1-\pi \beta<\cos (2 \pi \beta)=\sin (\pi / 2 \pm 2 \pi \beta)=\sin 2 \pi\left(\frac{1}{4} \pm \beta\right) \text {. }
$$

Thus, if

$$
(a, b) \in D_{\beta}\left(p_{0}^{+}\right) \subseteq\left[\frac{1}{4}-\beta, \frac{1}{4}+\beta\right] \times[1-\pi \beta, 1+\pi \beta]
$$

then

$$
s^{-1}(a, b) \in\left[\frac{1}{4}-\beta, \frac{1}{4}+\beta\right] \times[1-\pi \beta, 1+\pi \beta],
$$

because $s^{-1}$ takes vertical lines to vertical lines. Similarly, $\sin 2 \pi\left(-\frac{1}{4} \pm \beta\right)<$ $-1+\pi \beta$. Let $p=\left(p_{1}, p_{2}\right)$ and

$$
B=\left[p_{1}-\frac{1}{16 \pi}-\frac{\varepsilon}{\pi}, p_{1}+\frac{1}{16 \pi}+\frac{\varepsilon}{\pi}\right] \times\left[p_{2}-\frac{1}{16}-\varepsilon, p_{2}+\frac{1}{16}+\varepsilon\right] .
$$

Since $s^{-1} B \subseteq B, s^{-1}(q) \in B$. Let $s^{-1}(q)=\left(q_{1}, q_{2}\right)$. If $p=\left(\frac{1}{4}, 1\right)$,

$$
d\left(\sigma^{-1} s^{-1}(q), p\right)=\left(\left(q_{1}+q_{2}-1-\frac{1}{4}\right)^{2}+\left(q_{2}-1\right)^{2}\right)^{1 / 2} \leq\left(5\left(\frac{1}{16}+\varepsilon\right)^{2}\right)^{1 / 2}<\frac{3}{16} .
$$




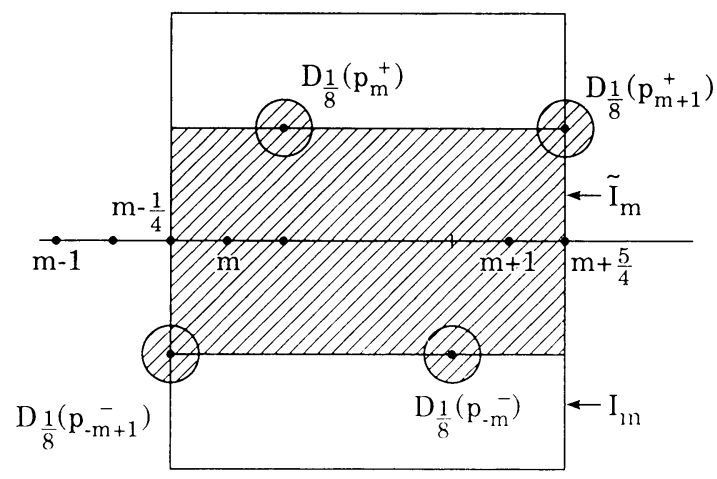

FIGURE 4

If $p=\left(\frac{3}{4},-1\right)$, a similar argument yields that $d\left(\sigma^{-1} s^{-1}(q), p\right)<\frac{3}{16}$. Then $W(q)=\alpha \sigma^{-1} s^{-1}(q) \in D_{1 / 64}(p)$ and $W_{1}(q) \in D_{1 / 16 \pi+\varepsilon / \pi}(p)$. Thus, we can continue: since $W_{1}(q) \in D_{1 / 16 \pi+\varepsilon / \pi}(p), W W_{1}(q) \in D_{1 / 64}(p)$, and $\widehat{W}_{2}(q) \in$ $D_{1 / 16 \pi+\varepsilon / \pi}(p)$. Eventually we obtain the result desired.

For each $m \in \mathscr{Z}$, let

$$
I_{m}=A_{0} \cap\left(\left[m-\frac{1}{4}, m+\frac{5}{4}\right] \times \mathbb{R}^{1}\right)
$$

and let

$\widetilde{I}_{m}=\left(\left[m-\frac{1}{4}, m+\frac{5}{4}\right] \times[-1,1]\right) \cup D_{1 / 8}\left(p_{m}^{+}\right) \cup D_{1 / 8}\left(p_{m+1}^{+}\right) \cup D_{1 / 8}\left(p_{-m}^{-}\right) \cup D_{1 / 8}\left(p_{-m+1}^{-}\right)$.

See Figure 4.

Lemma 2 (Space: $\mathbb{R}^{2}$ ). Suppose $0<\varepsilon<2^{-7} / \pi$. For each $m \in \mathscr{Z}, n \in \mathbb{N} \cup\{0\}$, $W\left[D_{\varepsilon}\left(\bigcup_{i=-n}^{n} \widetilde{I}_{m+i}\right)\right] \subseteq \bigcup_{i=-n-1}^{n+1} \widetilde{I}_{m+i}$. If for every $i \leq n, W_{i} \in N_{\varepsilon}(W)$, then $\widehat{W}_{n} \widetilde{I}_{m} \subseteq D_{\varepsilon}\left(\bigcup_{i=-n}^{n} \widetilde{I}_{m+i}\right)$.

Proof. For $n^{\prime} \in \mathbb{N} \cup\{0\}$, let $\theta_{n^{\prime}}=\left[m-\frac{1}{4}-n^{\prime}, m+\frac{5}{4}+n^{\prime}\right] \times[-1,1]$ and let

$$
\Gamma_{n^{\prime}}=\left(\bigcup_{i=-n^{\prime}}^{n^{\prime}+1} D_{1 / 8}\left(p_{m+i}^{+}\right)\right) \cup\left(\bigcup_{i=-n^{\prime}}^{n^{\prime}+1} D_{1 / 8}\left(p_{-m+i}^{-}\right)\right) \text {. }
$$

Note that $\bigcup_{i=-n^{\prime}}^{n^{\prime}} \widetilde{I}_{m+i}=\theta_{n^{\prime}} \cup \Gamma_{n^{\prime}}$.

Without loss of generality, suppose $m=0$. Suppose then that $(x, y) \in$ $D_{\varepsilon}\left(\bigcup_{i=-n}^{n} \widetilde{I}_{i}\right)$. If $W(x, y) \in \theta_{n+1}$, we are done, for $\theta_{n+1} \subseteq \bigcup_{i=-n-1}^{n+1} \widetilde{I}_{i}$. If $W(x, y) \notin \theta_{n+1}$, then $\sigma^{-1} s^{-1}(x, y)=(\hat{x}, \hat{y}) \notin \theta_{n+1}$, for otherwise $(\hat{x}, \hat{y}) \in$ $\theta_{n+1}$ and $\alpha\left(\theta_{n+1}\right)=\theta_{n+1}$. (Recall that $\alpha$ either does not move $(\hat{x}, \hat{y})$, or it moves it along a radial line $L$ containing some $p_{m+i}^{+}$or $p_{m+i}^{-}$and contained in $\theta_{n+1}$.)

Now $\sigma^{-1} s^{-1}(x, y)=(\hat{x}, \hat{y}) \notin \theta_{n+1}$ implies that $\hat{y}=\frac{1}{128} y+\frac{127}{128} \sin 2 \pi x>1$ or $\hat{y}<-1$, or $\hat{x}=x+\frac{1}{128} y+\frac{127}{128} \sin 2 \pi x>n+\frac{9}{4}$ or $\hat{x}<-n-\frac{5}{4}$. If $\hat{x}>n+\frac{9}{4}$, then $\hat{y}>\frac{7}{8}-\varepsilon$ (because $\hat{x}=x+\hat{y}$ and $x \leq n+\frac{5}{4}+\frac{1}{8}+\varepsilon$ ). Likewise, if $\hat{x}<-n-\frac{5}{4}$, then $\hat{y}<-\frac{7}{8}+\varepsilon$. Further, $x$ must be within $\frac{1}{8}$ unit from some $\pm \frac{1}{4}+j$, for otherwise $-\sqrt{2} / 2 \leq \sin 2 \pi x \leq \sqrt{2} / 2$ and $-\frac{7}{8}+\varepsilon<.83<\hat{y}=$ $\frac{1}{128} y+\frac{127}{128} \sin 2 \pi x<.83<\frac{7}{8}-\varepsilon$, which is impossible. If $\frac{7}{8}-\varepsilon<\hat{y} \leq 1$, then 
$\hat{x}=x+\hat{y}<n+\frac{9}{4}+\frac{1}{8}$, and $(\hat{x}, \hat{y}) \in \bigcup_{i=-n-1}^{n+1} \widetilde{I}_{i}$. Likewise, if $-1 \leq \hat{y}<-\frac{7}{8}+\varepsilon$, then $\hat{x}=x+\hat{y}>-n-\frac{1}{4}-1-\frac{1}{8}=-n-\frac{5}{4}-\frac{1}{8}$, and $(\hat{x}, \hat{y}) \in \bigcup_{i=-n-1}^{n+1} \widetilde{I}_{i}$. Suppose then that $\sin 2 \pi x>\sqrt{2} / 2$ and $\hat{y}>1$, or $\sin 2 \pi x<-\sqrt{2} / 2$ and $\hat{y}<-1$. Suppose first that $\sin 2 \pi x>\sqrt{2} / 2$ and $\hat{y}>1$. For some $p_{i}^{+}$, $\left|x-\pi_{1} p_{i}^{+}\right|<\frac{1}{8}$. Then

$$
\begin{aligned}
d^{2}\left(\sigma^{-1} s^{-1}(x, y), p_{i+1}^{+}\right) & \\
= & \left(x+\frac{1}{128} y+\frac{127}{128} \sin 2 \pi x-\left(i+\frac{5}{4}\right)\right)^{2} \\
& +\left(\frac{1}{128} y+\frac{127}{128} \sin 2 \pi x-1\right)^{2} \\
\leq & \left(x-\left(i+\frac{1}{4}\right)\right)^{2}+2\left|x-\left(i+\frac{1}{4}\right)\right|\left|\frac{1}{128} y+\frac{127}{128} \sin 2 \pi x-1\right| \\
& +2\left(\frac{1}{128} y+\frac{127}{128} \sin 2 \pi x-1\right)^{2} \\
< & \left(\frac{1}{8}\right)^{2}+2\left(\frac{1}{8}\right)\left(\frac{1}{128}+\frac{\varepsilon}{128}\right)+2\left(\frac{1}{128}+\frac{\varepsilon}{128}\right)^{2} \\
< & \frac{1}{64}+\frac{1}{4}\left(\frac{1}{128}+\frac{1}{2^{14}}\right)+2\left(\frac{1}{128}+\frac{1}{2^{14}}\right) \\
= & \frac{1}{64}+\left(\frac{1}{128}+2^{-14}\right)\left(\frac{9}{4}\right)<\left(\frac{3}{16}\right)^{2} .
\end{aligned}
$$

Thus, $d\left(\sigma^{-1} s^{-1}(x, y), p_{i+1}^{+}\right)<\frac{3}{16}$, and $d\left(W(x, y), p_{i+1}^{+}\right)<\frac{3}{16}\left(\left(\frac{3}{16}\right)^{3}+2^{-7}\right)<$ .05 . Then $W(x, y) \in \Gamma_{n+1}$. If $\sin 2 \pi x<-\sqrt{2} / 2$ and $\hat{y}<-1$, a similar proof yields the result $W(x, y) \in \Gamma_{n+1}$. Thus, we have proved the first statement.

Since $W\left(\widetilde{I}_{0}\right) \subseteq \bigcup_{i=-1}^{1} \widetilde{I}_{i}, W_{1}\left(\widetilde{I}_{0}\right) \subseteq D_{\varepsilon}\left(\bigcup_{i=-1}^{1} \widetilde{I}_{i}\right)$ and

$$
W W_{1}\left(\tilde{I}_{0}\right) \subseteq W\left(D_{\varepsilon}\left(\bigcup_{i=-1}^{I} \tilde{I}_{i}\right)\right) \subseteq \bigcup_{i=-2}^{2} \tilde{I}_{i} .
$$

Then $\widehat{W}_{2}\left(\widetilde{I}_{0}\right) \subseteq D_{\varepsilon}\left(\bigcup_{i=-2}^{2} \widetilde{I}_{i}\right)_{1}$ so that $W \widehat{W}_{2}\left(\widetilde{I}_{0}\right) \subseteq \bigcup_{i=-3}^{3} \widetilde{I}_{i}$. Continuing this process, we obtain the result.

Lemma 3 (Space: $S^{1} \times \mathbb{R}^{1}$ ). Suppose that $0<\varepsilon<2^{-7} / \pi, m$ is a positive number, and $\widetilde{T} \in C^{1}\left(S^{1} \times \mathbb{R}^{1}\right)$ with $\widetilde{T} \in N_{\varepsilon}^{1}(T)$. If $A \subseteq S^{1} \times \mathbb{R}^{1}, \frac{1}{2}>\operatorname{diam} \pi_{1} A$, and $\operatorname{diam} A>m$, then $\operatorname{diam} \widetilde{T}(A)>\sqrt{3} m$. If $A=[a, b] \times[c, d] \subseteq S^{1} \times \mathbb{R}^{1}$ with $b-a<1$ and $\operatorname{diam} A<m$, then $\widetilde{T}^{-1}(A)$ has two components $A_{1}$ and $A_{2}$, each of which maps onto $A$, and $\operatorname{diam} A_{i}<m / \sqrt{3}$ for $i=1,2$.

Proof. This follows from standard arguments and is omitted, although we remark that it is here that the differentiability of $\widetilde{T}$ is important. (If $\widetilde{T} \in$ $N_{\varepsilon}^{0}(T)-N_{\varepsilon}^{1}(T)$, then it is possible for $\widetilde{T}^{-1}(A)$ (for $A=[a, b] \times[c, d]$, $b-a<1)$ to have more than two components. Further, if $\operatorname{diam} A<m<\varepsilon$, it is possible that $\operatorname{diam} \widetilde{A} \geq m / \sqrt{3}$. for some component $\widetilde{A}$ of $\widetilde{T}^{-1}(A)$.) 
Lemma 4. One-component, three-component property (Space: $\mathbb{R}^{2}$ ). Suppose $0<$ $\varepsilon<1 / 128 \pi, k \in \mathbb{N}, n \in \mathscr{Z}$, and $\frac{3}{2}>\delta>\frac{9}{8}+\varepsilon$. If for each $i \in\{1, \ldots, k\}$, $W_{i} \in N_{\varepsilon}^{1}(W)$, then $\widehat{W}_{k}\left(A_{0}\right)$ has the following properties:

(1) One-component property. $\widehat{W}_{k}\left(A_{0}\right) \cap\left(\left[\pi_{1} p_{-n+k+1}^{-}, \pi_{1} p_{n+k+1}^{+}\right] \times \mathbb{R}^{1}\right)$ has exactly one component $E$ that intersects both $\left(D_{1 / 16 \pi+\varepsilon}\left(\pi_{1} p_{n+k+1}^{+}\right) \times \mathbb{R}^{1}\right)$ and $\left(D_{1 / 16+\varepsilon}\left(\pi_{1} p_{-n+k+1}^{-}\right) \times \mathbb{R}^{1}\right)$.

(2) Three-component property. $\widehat{W}_{k}\left(A_{0}\right) \cap\left(\left[\pi_{1} p_{-n+k+1}^{-}+\delta, \pi_{1} p_{n+k+1}^{+}-\delta\right] \times \mathbb{R}^{1}\right)$ and $E \cap\left(\left[\pi_{1} p_{-n+k+1}^{-}+\delta, \pi_{1} p_{n+k+1}^{+}-\delta\right] \times \mathbb{R}^{1}\right)$ each have exactly three components that intersect both $\left\{\pi_{1} p_{-n+k+1}^{-}+\delta\right\} \times \mathbb{R}^{1}$ and $\left\{\pi_{1} p_{n+k+1}^{+}-\right.$ $\delta\} \times \mathbb{R}^{1}$.

Proof. We proceed by induction. Let $k=1$. Fix $n$. If $L$ is a horizontal interval at height between -2 and 2 and extending from $\pi_{1} p_{-n+1}^{-}$to $\pi_{1} p_{n+1}^{+}$, then $W(L)$ is a path beginning within $\frac{1}{64}$ of the point $p_{-n+2}^{-}$; then increasing from there to within $\frac{1}{64}$ of $p_{n+1}^{+}$; then decreasing, with respect to both vertical and horizontal directions, to within $\frac{1}{64}$ of $p_{-n+1}^{-}$; and finally increasing, with respect to both directions, to within $\frac{1}{64}$ of $p_{n+2}^{+}$. Since $W_{1} \in N_{\varepsilon}^{1}(W), W_{1}(L)$ is within $\frac{1}{128 \pi}$ of $W(L)$. The path $W_{1}(L)$ does not extend beyond $1 / 64+1 / 128 \pi$ of any point on the vertical line through $\left(n+\frac{9}{4}, 1\right)$ nor does it extend beyond $1 / 64+1 / 128 \pi$ of any point on the vertical line through $\left(n-\frac{5}{4},-1\right)$, and it only comes close to the points $\left(n+\frac{9}{4}, 1\right)$ and $\left(n-\frac{5}{4},-1\right)$ on these respective vertical lines. (Lemmas 1 and 2 guarantee these results. See Figure 5.)

For $n \in \mathbb{Z}$, let $A_{0}^{n}=\left[n-\frac{1}{4}, n+\frac{5}{4}\right] \times[-2,2]$. Then $W_{1}\left(A_{0}^{n}\right)$ consists of a continuous collection of paths beginning close to $p_{-n+2}^{-}$, increasing almost to $p_{n+1}^{+}$, decreasing almost to $p_{-n+1}^{-}$and increasing almost to $p_{n+2}^{+}$, and because of Lemmas 1 and 2, the result follows for $W_{1}\left(A_{0}^{n}\right)$. The result then follows easily for $W_{1}\left(A_{0}\right)=W_{1}\left(\bigcup_{n \in \mathscr{Z}} A_{0}^{n}\right)$.

Suppose that the lemma holds for $k-1$ and each $n \in \mathscr{Z}$. Then

(1) $\widehat{W}_{k-1}\left(A_{0}\right) \cap\left[\left(\pi_{1} p_{-n+k}^{-}, \pi_{1} p_{n+k}^{+}\right] \times \mathbb{R}^{1}\right)$ has exactly one component $E_{k-1}$ that intersects both $\left(D_{1 / 16 \pi+\varepsilon}\left(\pi_{1} p_{n+k}^{+}\right) \times \mathbb{R}^{1}\right)$ and $\left(D_{1 / 16 \pi+\varepsilon}\left(\pi_{1} p_{-n+k}^{-}\right) \times \mathbb{R}^{1}\right)$, and

(2) $\widehat{W}_{k-1}\left(A_{0}\right) \cap\left(\left[\pi_{1} p_{-n+k}^{-}+\delta, \pi_{1} p_{n+k}^{+}-\delta\right] \times \mathbb{R}^{1}\right)$ and $E_{k-1} \cap\left(\left[\pi_{1} p_{-n+k}^{-}+\right.\right.$ $\left.\left.\delta, \pi_{1} p_{n+k}^{+}-\delta\right] \times \mathbb{R}^{1}\right)$ each contain exactly three components that intersect both $\left\{\pi_{1} p_{-n+k}^{-}+\delta\right\} \times \mathbb{R}^{1}$ and $\left\{\pi_{1} p_{n+k}^{+}-\delta\right\} \times \mathbb{R}^{1}$.

Consider $\widehat{W}_{k}\left(A_{0}\right)=W_{k}\left(\widehat{W}_{k-1}\left(A_{0}\right)\right)$. Fix $n$. If $L$ is a horizontal interval at height between -2 and 2 and extending from within $1 / 16 \pi+\varepsilon$ of $\pi_{1} p_{-n+k}^{-}$to within $1 / 16 \pi+\varepsilon$ of $\pi_{1} p_{n+k}^{+}$, then $W_{k}(L)$ is a path whose left most endpoint is within $1 / 16 \pi+\varepsilon$ of $p_{-n+k+1}^{-}$and whose rightmost endpoint is within $1 / 16 \pi+\varepsilon$ of $p_{n+k+1}^{+}$. From its left endpoint, the path $W_{k}(L)$ increases (with respect to both the $x$ and $y$ directions) to within $1 / 16 \pi+\varepsilon$ of $p_{n-k+2}^{+}$; decreases (both $x$ and $y$ directions) to within $1 / 16 \pi+\varepsilon$ of $p_{-n+k}^{-}$; increases to within $1 / 16 \pi+\varepsilon$ of $p_{n-k+3}^{+} ; \ldots$; decreases from within $1 / 16 \pi+\varepsilon$ of $p_{n+k}^{+}$to within $1 / 16 \pi+\varepsilon$ of $p_{-n-k-2}^{-}$; and finally increases from within $1 / 16 \pi+\varepsilon p_{-n-k+2}^{-}$to within $1 / 16 \pi+\varepsilon$ of $p_{n+k+1}^{+}$. Then $W_{k}\left(\left[\pi_{1} p_{-n+k}^{-}, \pi_{1} p_{+n+k}^{+}\right] \times[-2,2]\right)$ is a continuous collection of paths increasing and decreasing in the same manner from within 
$1 / 16 \pi+\varepsilon$ of $p_{-n+k+1}^{-}$to within $1 / 16 \pi+\varepsilon$ of $p_{n+k+1}^{+}$, because of Lemmas 1 and 2. Also because of Lemmas 1 and 2, $\widehat{W}_{k}\left(A_{0}\right) \cap\left(\left[\pi_{1} p_{-n+k+1}^{-}, \pi_{1} p_{n+k+1}^{+}\right] \times\right.$ $\left.\mathbb{R}^{1}\right) \subseteq W_{k}\left(\left[\pi_{1} p_{-n+k}^{-}, \pi_{1} p_{n+k}^{+}\right] \times[-2,2]\right)$ extends from within $1 / 16 \pi+\varepsilon$ of $p_{-n+k+1}^{-}$to within $1 / 16 \pi+\varepsilon$ of $p_{n+k+1}^{+}$, because $E_{k-1}$, the "main" component of $\widehat{W}_{k-1}\left(A_{0}\right)$ extends from within $1 / 16 \pi+\varepsilon$ of $p_{-n+k}^{-}$to within $1 / 16 \pi+\varepsilon$ of $p_{n+k}^{+}$. Further, if the three components of $E_{k-1} \cap\left(\left[\pi_{1} p_{-n+k}^{-}+\delta, \pi_{1} p_{n+k}^{+}-\delta\right] \times \mathbb{R}^{1}\right)$ that intersect both vertical lines $x=\pi_{1} p_{-n+k}^{-}+\delta$ and $x=\pi_{1} p_{n+k}^{+}-\delta$ are denoted $E_{k-1}^{1}, E_{k-1}^{2}, E_{k-1}^{3}$, then

$$
\begin{gathered}
W_{k}\left(E_{k-1}\right) \cap\left(\left[\pi_{1} p_{-n+k+1}^{-}+\delta, \pi_{1} p_{n+k+1}^{+}-\delta\right] \times \mathbb{R}^{1}\right) \\
\supseteq\left[W_{k}\left(E_{k-1}^{1}\right) \cup W_{k}\left(E_{k-1}^{2}\right) \cup W_{k}\left(E_{k-1}^{3}\right)\right] \\
\cap\left(\left[\pi_{1} p_{-n+k+1}^{-}+\delta, \pi_{1} p_{n+k+1}^{+}-\delta\right] \times \mathbb{R}^{1}\right),
\end{gathered}
$$

and each $E_{k}^{i}=W_{k}\left(E_{k-1}^{i}\right) \cap\left(\left[\pi_{1} p_{-n+k+1}^{-}+\delta, \pi_{1} p_{n+k+1}^{+}-\delta\right] \times \mathbb{R}^{1}\right)$ is a continuum intersecting both vertical lines $x=\pi_{1} p_{-n+k+1}^{-}+\delta$ and $x=\pi_{1} p_{-n+k+1}^{-}+\delta$. Thus, $E_{k}^{1}, E_{k}^{2}$, and $E_{k}^{3}$ are the three components of the Lemma. See Figure 5.

Lemma $4^{\prime}$. One-component, three-or-more-component property (Space: $\mathbb{R}^{2}$ ). Suppose $0<\varepsilon<1 / 128 \pi, k \in \mathbb{N}, n \in \mathscr{Z}, m \in \mathbb{N}$, and $m \leq k+1$, and $\frac{3}{2}>\delta>\frac{9}{8}+\varepsilon$. If for each $i \in\{1, \ldots, k\}, W_{i} \in N_{\varepsilon}^{1}(W)$, then $\widehat{W}_{k}\left(A_{0}\right) \cap$ $\left(\left[\pi_{1} p_{-n+m}^{-}, \pi_{1} p_{n+m}^{+}\right] \times \mathbb{R}^{1}\right)=D$ has the following properties:

(1) One-component property. $D$ has a least one component $E$ that intersects both $\left(D_{1 / 16 \pi+\varepsilon}\left(\pi_{1} p_{n+m}^{+}\right) \times \mathbb{R}^{1}\right)$ and $\left(D_{1 / 16 \pi+\varepsilon}\left(\pi_{1} p_{-n+m}^{-}\right) \times \mathbb{R}^{1}\right)$.

(2) Three-component (or more) property. $E \cap\left(\left[\pi_{1} p_{-n+m}^{-}+\delta, \pi_{1} p_{n+m}^{+}\right] \times \mathbb{R}^{1}\right)$, where $E$ is a component as in (1), contains at least three components intersecting both the vertical lines through $\pi_{1} p_{-n+m}^{-}+\delta$ and $\pi_{1} p_{n+m}^{+}-\delta$.

Proof. This lemma is a slight generalization of Lemma 4, and its proof is similar. It is therefore omitted.

Theorem 5. There is a sequence $g_{1}, g_{2}, \ldots$ of maps on $S^{1} \times \mathbb{R}^{1}$ such that

(1) for each $i, g_{i} \in N_{\varepsilon}^{1}\left(W^{-1}\right) \cup N_{\varepsilon}^{1}(T)$, and

(2) $\bigcap_{i=0}^{\infty}\left(\hat{g}_{i}^{-1}\right)\left(A_{0}\right)$ is pseudocircle that separates $S^{1} \times \mathbb{R}^{1}$ into two open sets, each homeomorphic to $S^{1} \times \mathbb{R}^{1}$.

Proof. Choose $m_{1} \in \mathbb{N}$ so that if $\left\{T_{11}, T_{12}, \ldots, T_{1, m_{1}}\right\}$ is contained in $N_{\varepsilon}^{1}(T)$, then each component of $\widehat{T_{1, m_{1}}^{-1}}\left(\left(-\frac{1}{4}, \frac{3}{4}\right) \times[-2,2]\right)$ had diameter less than $\frac{1}{2}$. Choose an $m_{1}$-element subset $\left\{T_{1,1}, \ldots, T_{1, m_{1}}\right\}$ of $N_{\varepsilon}^{1}(T)$. The collection of closures of components $\left.\left(\widehat{T_{1, m_{1}}^{-1}}\right)\left(\left(-\frac{1}{4}, \frac{3}{4}\right) \times[-2,2]\right)\right)$ forms a circular chain cover $C_{1}=\left\{c(1,1), \ldots, c\left(1, p_{1}\right)\right\}$ of $\left(\widehat{T_{1, m_{1}}^{-1}}\left(A_{0}\right)\right.$ of mesh less than $\frac{1}{2}$. Let $F_{1}=$ $T_{11} \circ \cdots \circ T_{1, m_{1}}$. For each $j, 1 \leq j \leq p_{1}, F_{1}(c(1, j))=A_{0}=\left[-\frac{1}{4}, \frac{3}{4}\right] \times[-2,2]$.

Now switch the setting from $\mathbb{R}^{1} \times S^{1}$ to $\mathbb{R}^{2}$, i.e., go to the lift of $F_{1}$ and covering space of $\mathbb{R}^{1} \times S^{1}$. In this setting, we can think of $F_{1}(c(1, j))$ as being $\left[-\frac{1}{4}+j-1, \frac{3}{4}+j-1\right] \times[-2,2]$. If $p_{1} \leq 5$, let $n_{1}=0$. Otherwise 


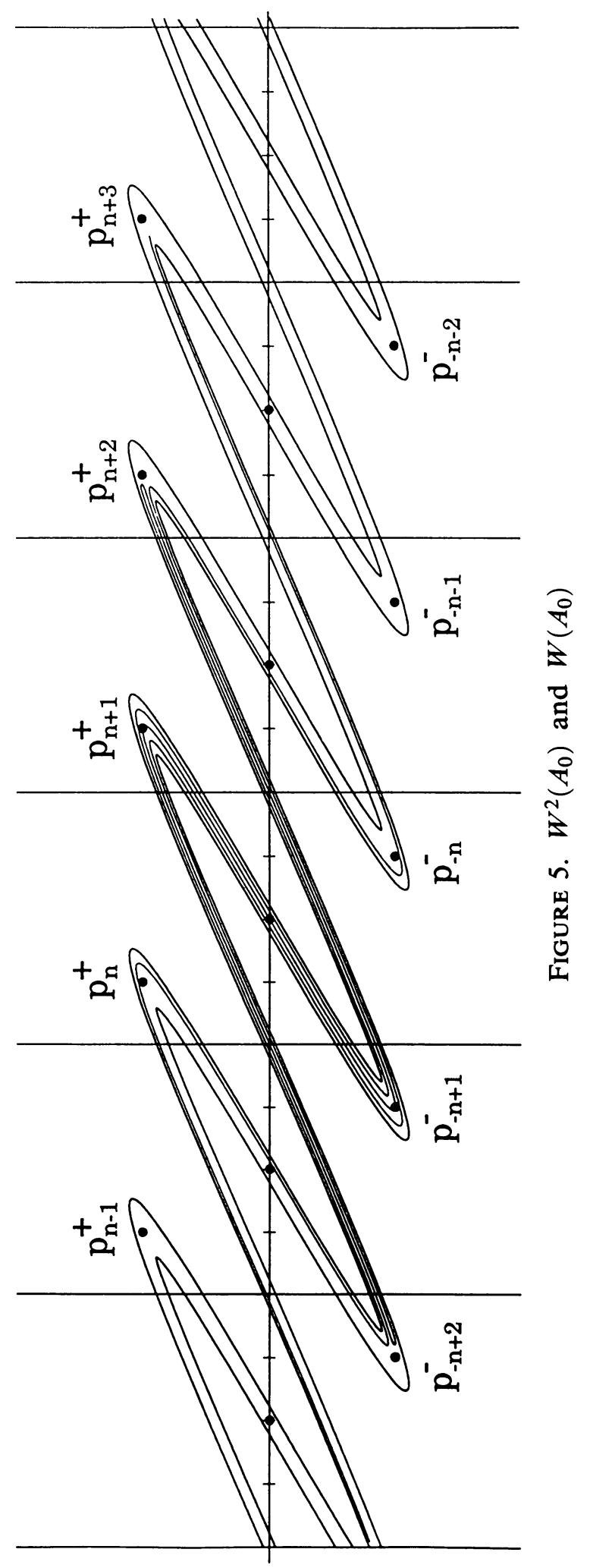


choose $n_{1}$ to be an integer such that $n_{1}>p_{1} / 2$. Choose an $n_{1}$-element subset $\left\{W_{1,1}, \ldots, W_{1, n_{1}}\right\}$ of $N_{\varepsilon}^{1}(W)$.

Switch the setting back to $\mathbb{R}^{1} \times S^{1}$. Let $A_{1}=\left(\widehat{T_{1, m_{1}}^{-1}}\right) \circ \widehat{W_{1, n_{1}}}\left(A_{0}\right)$. Then $A_{1} \subseteq A_{0}$ and $A_{1}$ is covered by the circular chain $C_{1}$. (Recall that with respect to $\mathbb{R}^{2}$ and the lift of $F_{1}$, also referred to as $F_{1}, F_{1}(c(1, j))=\left[-\frac{1}{4}+j-\right.$ $\left.1, \frac{3}{4}+j-1\right] \times[-2,2]$ for $1 \leq j \leq p_{1}$. Choosing $n_{1}>p_{1} / 2$ ensures that $\left(\widehat{W}_{1, n_{1}}\right)^{-1}\left(\left[-\frac{1}{4}, p_{1}-\frac{1}{4}\right] \times[-2,2]\right)=D_{1}$ has the one-component-three- (or more) component property of Lemma $4^{\prime}$ with respect to $D_{0}=\left[-\frac{1}{4}, p_{1}-\frac{1}{4}\right] \times$ $[-2,2]$. That is, there is at least one component of $D_{0} \cap D_{1}$ that comes within $1 / 16 \pi+\varepsilon$ of the vertical lines through $-\frac{1}{4}$ and $p_{1}-\frac{1}{4}$, and if $\frac{3}{2}>\delta>\frac{9}{8}+\varepsilon$ and $E$ is such a component then $E \cap\left(\left[-\frac{1}{4}+\delta, p_{1}-\frac{1}{4}-\delta\right] \times[-2,2]\right)$ contains at least three components that intersect both the vertical lines $x=-\frac{1}{4}+\delta$ and $x=p_{1}-$ $\frac{1}{4}-\delta$. Thus, we have the one-component-three- (or more) component property for $D_{1}$ in $D_{0}$ and for $A_{1}$ in $\left.A_{0}.\right)$ Let $G_{1}=W_{1,1}^{-1} \circ \cdots \circ W_{1, n_{1}}^{-1} \circ T_{1,1} \circ \cdots \circ T_{1, m_{1}}$. Choose $m_{2} \in \mathbb{N}$ so that if $\left\{T_{2,1}, \ldots, T_{2}, m_{1}\right\} \subseteq N_{\varepsilon}^{1}(T)$, then each component of $\left(\widehat{T_{1, m_{1}}^{-1}}\right) \circ \widehat{W_{1, n_{1}}} \circ\left(\widehat{T_{2, m_{2}}^{-1}}\right)\left(\left(-\frac{1}{4}, \frac{3}{4}\right) \times[-2,2]\right)$ has diameter less than $\frac{1}{4}$. The collection of closures of components of $\left(\widehat{T_{1, m_{1}}^{-1}}\right) \circ \widehat{W_{1, n_{1}}} \circ\left(\widehat{T_{2, m_{2}}^{-1}}\right)\left(\left(-\frac{1}{4}, \frac{3}{4}\right) \times\right.$ $[-2,2])$ forms a circular chain cover $C_{2}=\left\{c(2,1), \ldots, c\left(2, p_{2}\right)\right\}$ of $\widehat{A_{2}}=$ $\left(\widehat{T_{1, m_{1}}^{-1}}\right) \circ\left(\widehat{W_{1, n_{1}}}\right) \circ\left(\widehat{T_{2, m_{2}}^{-1}}\right)\left(A_{0}\right)$ of mesh less than $\frac{1}{4}$. Let $F_{2}=T_{21} \circ \cdots \circ T_{2, m_{2}} \circ$ $W_{1,1}^{-1} \circ \cdots \circ W_{1, n_{1}}^{-1} \circ T_{1,1} \circ \cdots \circ T_{1, m_{1}}$. For each $j, 1 \leq j \leq p_{2}, F_{2}(c(2, j))=A_{0}$. Choose $n_{2}$ to be an integer $>p_{2} / 2$. (Again, this is to satisfy Lemma $4^{\prime}$ for $A_{2}$ relative to $\widehat{A_{2}}$.) Choose an $n_{2}$-element subset $\left\{W_{2,1}, \ldots, W_{2, n_{2}}\right\}$ of $N_{\varepsilon}(W)$. Let $A_{2}=\left(\widehat{T_{1, m_{1}}^{-1}}\right) \circ\left(\widehat{W_{1, n_{1}}}\right) \circ\left(\widehat{T_{2, m_{2}}^{-1}}\right) \circ\left(\widehat{W_{2, m_{2}}}\right)\left(A_{0}\right)$.

Continue this process. At the ith level, $A_{i}=\left(\widehat{T_{1, m_{1}}^{-1}}\right) \circ \widehat{W_{1, n_{1}}} \circ\left(\widehat{T_{2, m_{2}}^{-1}}\right) \circ$ ... $\left(\widehat{T_{i, m_{i}}^{-1}}\right) \circ \widehat{W_{i, n_{i}}}\left(A_{0}\right) \subset A_{i-1}$, with $A_{i}$ covered by the circular chain cover $C_{i}=\left\{c(i, 1), \ldots, c\left(i, p_{i}\right)\right\}$, the collection of closures of components of $\widehat{A_{i}}=$ $\left(\widehat{T_{1, m_{1}}^{-1}}\right) \circ\left(\widehat{W_{1, n_{1}}}\right) \circ \cdots \circ\left(\widehat{T_{i, m_{i}}^{-1}}\right)\left(A_{0}\right)$, and $m_{i}$ chosen so that mesh $C_{i}<2^{-i}$. Then choose $n_{i}$ to be an integer $>p_{i} / 2$, and choose $\left\{W_{i, 1}, \ldots, W_{i, n_{i}}\right\}$ to be an an $n_{i}$-element subset of $N_{\varepsilon}^{1}(W)$.

Note that $A_{0} \supseteq A_{1} \supseteq \cdots$ and $X=\bigcap_{i=0}^{\infty} A_{i}$ is a continuum that separates $S^{1} \times \mathbb{R}^{1}$ into two open sets, each homeomorphic to $S^{1} \times \mathbb{R}^{1}$. Since for each $i, C_{i}$ is a circular chain that covers $X$ and has mesh less than $1 / 2^{i}, X$ is circlelike.

We need to show that $X$ is hereditarily indecomposable. To this end, suppose that $H$ and $K$ are disjoint, closed subsets of $X$ and that $U$ and $V$ are open sets in $S^{1} \times \mathbb{R}^{1}$ such that $H \subseteq U, K \subset V$, and $\bar{U} \cap \bar{V}=\varnothing$. There is some $i$ such that $d(H, X-U)>2^{4-i}, d(K, X-V)>2^{4-i}$, and $d(\bar{U}, \bar{V})>2^{4-i}$.

Consider $C_{i}$. Construct the sets $X_{0}, X_{1}$, and $X_{2}$ as follows:

(1) If $c(i, j) \cap H \neq \varnothing$, then $c(i, j) \subseteq X_{0}$.

(2) If $c(i, j) \cap K \neq \varnothing$, then $c(i, j) \subseteq X_{2}$.

(3) If $C^{\prime}$ is a subchain of $C_{i}$ such that both end links of $C^{\prime}$ intersect $H$, but no link of $C^{\prime}$ intersects $K$, then $\cup C^{\prime} \subseteq X_{0}$.

(4) If $C^{\prime}$ is a subchain of $C_{i}$ such that both end links of $C^{\prime}$ intersect $K$, but no link of $C^{\prime}$ intersects $H$, then $\bigcup C^{\prime} \subseteq X_{2}$. 


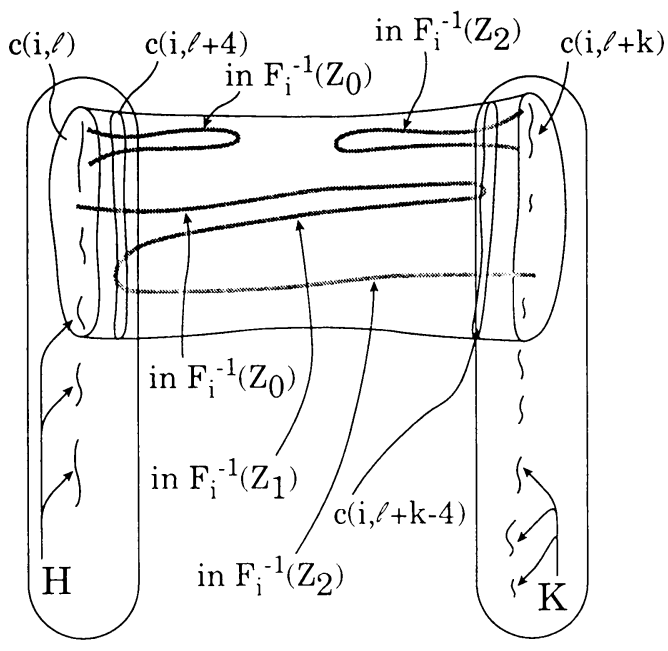

FIGURE 6

(5) Suppose $C^{\prime}$ is a subchain of $C_{i}$ such that one end link of $C^{\prime}$ intersects $H$, the other end link intersects $K$, and no other link of $C^{\prime}$ intersects either $H$ or $K$. Without loss of generality, let us say that $C^{\prime}=\{c(i, l), \ldots, c(i, l+k)\}$, $c(i, l) \cap H \neq \varnothing$, and $c(i, l+k) \cap K \neq \varnothing$. There is $j, 0 \leq j \leq n_{i}-1$, such that $Z=W_{i, n_{i}} \circ \cdots \circ W_{i, n_{i}-j}\left(A_{0}\right) \cap F_{i}\left(\cup C^{\prime}\right)$ has exactly one component (in $\left.\mathbb{R}^{2}\right)$ that intersects both $F_{i}(c(i, l))$ and $F_{i}(c(i, l+k))$, and if $C^{\prime \prime}=$ $\{c(i, l+4), \ldots, c(i, l+k-4)\}$, then $Z \cap F_{i}\left(\cup C^{\prime \prime}\right)=Z^{\prime}$ has at least three components that intersect both $F_{i}(c(i, l+4))$ and $F_{i}(c(i, l+k-4))$. (See Figure 6. We are applying Lemma 4 here and using the one-component-threecomponent property it gives to get the "zigzag" needed between $H$ and $K$ to satisfy part III of the Krasiniewicz-Minc Theorem. One appropriate way to choose the " $n$ " of Lemma 4 is to let $n$ denote the greatest integer less than or equal to $k / 2$, and then $j+1$ is the " $k$ " of Lemma 4 , and $W_{i, n_{i}-j}$ corresponds to " $W_{1}$ " in Lemma $4, W_{1, n_{i}-j+1}$ to " $W_{2}$ ", $\ldots$ and $W_{i, n_{i}}$ to " $W_{k}$ ".) Construct sets $Z_{0}, Z_{1}$, and $Z_{2}$ as follows:

(a) If $L$ is a component of $Z$ intersecting $F_{i}(c(i, l))$, but not $F_{i}(c(i, l+k))$, then $L \subseteq Z_{0}$.

(b) If $L$ is a component of $Z$ intersecting $F_{i}(c(i, l+k))$, but not $F_{i}(c(i, l))$, then $L \subseteq Z_{2}$.

(c) If $L$ is the component of $Z$ intersecting both $F_{i}(c(i, l))$ and $F_{i}(c(i, l+k))$, then because of the "three component property" of $Z^{\prime}, L$ can be naturally divided into closed sets $L_{0}, L_{1}, L_{2}$ such that $L_{0} \cap L_{1} \subseteq F_{i}\left(\bigcup_{\hat{k}=k-4}^{k} c(i, l+\hat{k})\right)$, $L_{1} \cap L_{2} \subseteq F_{i}\left(\bigcup_{\hat{k}=0}^{4} c(i, l+\hat{k})\right), \quad L_{0} \cap L_{2}=\varnothing, \quad F_{i}(c(i, l)) \cap L \subseteq L_{0}$, $F_{i}(c(i, l+k)) \cap L \subseteq L_{2}$, and $L_{0} \cup L_{1} \cup L_{2}=L$. Then $L_{0} \subseteq Z_{0}, L_{1}=Z_{1}$, and $L_{2} \subseteq Z_{2}$.

Finally, $F_{i}^{-1}\left(Z_{0}\right) \subseteq X_{0}, F_{i}^{-1}\left(Z_{1}\right) \subseteq X_{1}$, and $F_{i}^{-1}\left(Z_{2}\right) \subseteq X_{2}$. Then $X_{0}, X_{1}$, and $X_{2}$ are closed sets such that

(6) $X_{0} \cup X_{1} \cup X_{2} \supseteq X$,

(7) $X_{0} \cap X_{1} \subseteq V$,

(8) $X_{1} \cap X_{2} \subseteq U$,

(9) $H \subseteq X_{0}$ and $K \subseteq X_{2}$, and 
(10) $X_{0} \cap X_{2}=\varnothing$.

Thus by the Krasinkiewicz-Minc Theorem, $X$ is hereditarily indecomposable. Since nondegenerate, hereditarily indecomposable, circularly chainable continua in $\mathbb{R}^{2}$ (or $S^{1} \times \mathbb{R}^{1}$ ) are pseudocircles, $X$ is a pseudocircle.

We define a washer to be a set homeomorphic to $S^{1} \times[0,1] \times[0,1]$. Thus, a washer is just a set homeomorphic to a solid torus.

Theorem 6. There are a $C^{1}$-map $F_{0}: \mathbb{R}^{3} \rightarrow \mathbb{R}^{3}, \varepsilon>0$, and disjoint copies $R_{1}$ and $R_{2}$ of $S^{1} \times I \times I$ such that if $F \in N_{\varepsilon}^{1}\left(F_{0}\right)$, then

(1) $\left(F\left(R_{1}\right)\right)^{\circ} \supseteq R_{1} \cup R_{2}$ and $\left(F\left(R_{2}\right)\right)^{\circ} \supseteq R_{1} \cup R_{2}$,

(2) $F$ is a local diffeomorphism on $R_{1} \cup R_{2}$ and is differentiable on $\mathbb{R}^{3}$,

(3) $\mathbb{B}=\left\{(x, y, z) \mid F^{n}(x, y, z) \in R_{1} \cup R_{2}\right.$ for each $\left.n \geq 0\right\}$ is a continuous Cantor set of continua with $F(\mathbb{B})=\mathbb{B}$, and

(4) there is a dense $G_{\delta}$-subset $G$ of $\mathbb{B}$ such that $G$ is a union of components of $\mathbb{B}$ and each component of $G$ is a pseudocircle.

Proof. Let $I_{1}$ and $I_{2}$ be disjoint intervals in $S^{1}$ with $I_{1}=\left[-\frac{1}{128}, \frac{41}{128}\right]$ and $I_{2}=\left[\frac{63}{128}, \frac{100}{128}\right]$. Note that $\frac{1}{4}<\left|I_{1}\right|<\frac{1}{3}$ and $\frac{1}{4}<\left|I_{2}\right|<\frac{1}{3}$. Let $R_{1}=S^{1} \times$ $[-2,2] \times I_{1}$ and $R_{2}=S^{1} \times[-2,2] \times I_{2}$.

For $z \in S^{1}$, let $g(z)=3 z \bmod 1$. Then $g \mid I_{1}$ and $g \mid I_{2}$ are one-to-one maps with $g\left(I_{1}^{0}\right)$ and $g\left(I_{2}^{0}\right)$ each containing both $I_{1}$ and $I_{2}$. A standard argument then gives that if $\alpha_{0}, \alpha_{1}, \ldots$ is a sequence of 1's and 2's, then there exists exactly one $z$ in $S^{1}$ such that $g^{i}(z) \in I_{\alpha_{i}}$ for each $i \in \widetilde{\mathbb{N}}$. Further, $C=\left\{z \in S^{1} \mid g^{n}(z) \in I_{1} \cup I_{2}\right.$ for each $\left.n \in \widetilde{\mathbb{N}}\right\}$ is a Cantor set in $S^{1}$.

Define $F_{0}: S^{1} \times \mathbb{R}^{1} \times\left(I_{1} \cup I_{2}\right) \rightarrow S^{1} \times \mathbb{R}^{1} \times S^{1}$ by $F_{0}(x, y, z)=(T(x, y), g(z))$ if $z \in I_{1}$ and $F_{0}(x, y, z)=\left(W^{-1}(x, y), g(z)\right)$ if $z \in I_{2}$. Note that $F_{1}=$ $F_{0} \mid S^{1} \times \mathbb{R}^{1} \times I_{1}$ is a local diffeomorphism, while $F_{2}=F_{0} \mid S^{1} \times \mathbb{R}^{1} \times I_{2}$ is a diffeomorphism. Choose $\varepsilon>0, \varepsilon<2^{-9} / \pi$, so that if $F \in N_{\varepsilon}^{1}\left(F_{0}\right)$, then (1) $F^{-1} \mid F\left(S^{1} \times \mathbb{R}^{1} \times I_{2}\right) \in N_{2^{-7} / \pi}^{1}\left(F_{2}^{-1}\right)$ and (2) if $\theta \subseteq F\left(S^{1} \times[-2,2] \times I_{1}\right)$ with $\operatorname{diam} \theta<\frac{1}{2}$, then $F^{-1} \mid \theta \in N_{2^{-7} / \pi}^{1}\left(F_{1}^{-1} \mid \theta\right)$. (If $\theta$ is connected, then both $F_{1}^{-1} \mid \theta$ and $F^{-1} \mid \theta$ have exactly two components, each mapped homeomorphically onto $\theta$ by $F_{1}$ and $F$.)

Suppose $F \in N_{\varepsilon}^{1}\left(F_{0}\right)$. Checking that $\left(F\left(R_{1}\right)\right)^{\circ} \supseteq R_{1} \cup R_{2}$ and $\left(F\left(R_{2}\right)\right)^{\circ} \supseteq$ $R_{1} \cup R_{2}$ is straightforward. Also, since $F$ is in $N_{\varepsilon}^{1}\left(F_{0}\right), F$ is a local diffeomorphism on $R_{1} \cup R_{2}$.

Remember that $F_{0}$ and $F$ have been defined only on $S^{1} \times \mathbb{R}^{1} \times\left(I_{1} \cup I_{2}\right)$ so far. We can visualize $F_{0}^{-1}\left(R_{1}\right)$ and $F_{0}^{-1}\left(R_{2}\right)$ as follows: Each $(x, y, z)$ in $R_{1}$ has exactly three preimages in $S^{1} \times \mathbb{R}^{1} \times\left(I_{1} \cup I_{2}\right)$. It has two preimages in $R_{1}$ and one in $R_{2}$. The two preimages in $R_{1}$ occur because $T$ is two-to-one and $\left(\frac{1}{2} x, \frac{1}{2} y\right)$ actually gives two points. The third coordinate $z / 3$ is unique, so $\left(\frac{1}{2} x, \frac{1}{2} y, \frac{1}{3} z\right)=\left(T^{-1}(x, y), g^{-1}(z)\right)$ corresponds to two points. There is only one preimage $\left(W(x, y), g^{-1}(z)\right)$, since $W$ is a diffeomorphism and $g \mid I_{2}$ is one-to-one.

The set $F_{0}^{-1}\left(R_{1}\right)$ consists of two components, one component being in $R_{1}$ and the other in $R_{2}$. Under $F_{0}$ slices in the $z$-direction get moved to slices in the $z$-direction.

The component of $F_{0}^{-1}\left(R_{1}\right)$ in $R_{1}$ is the set $S^{1} \times[-1,1] \times\left[-\frac{1}{3 \cdot 128}, \frac{41}{3 \cdot 128}\right]$, 


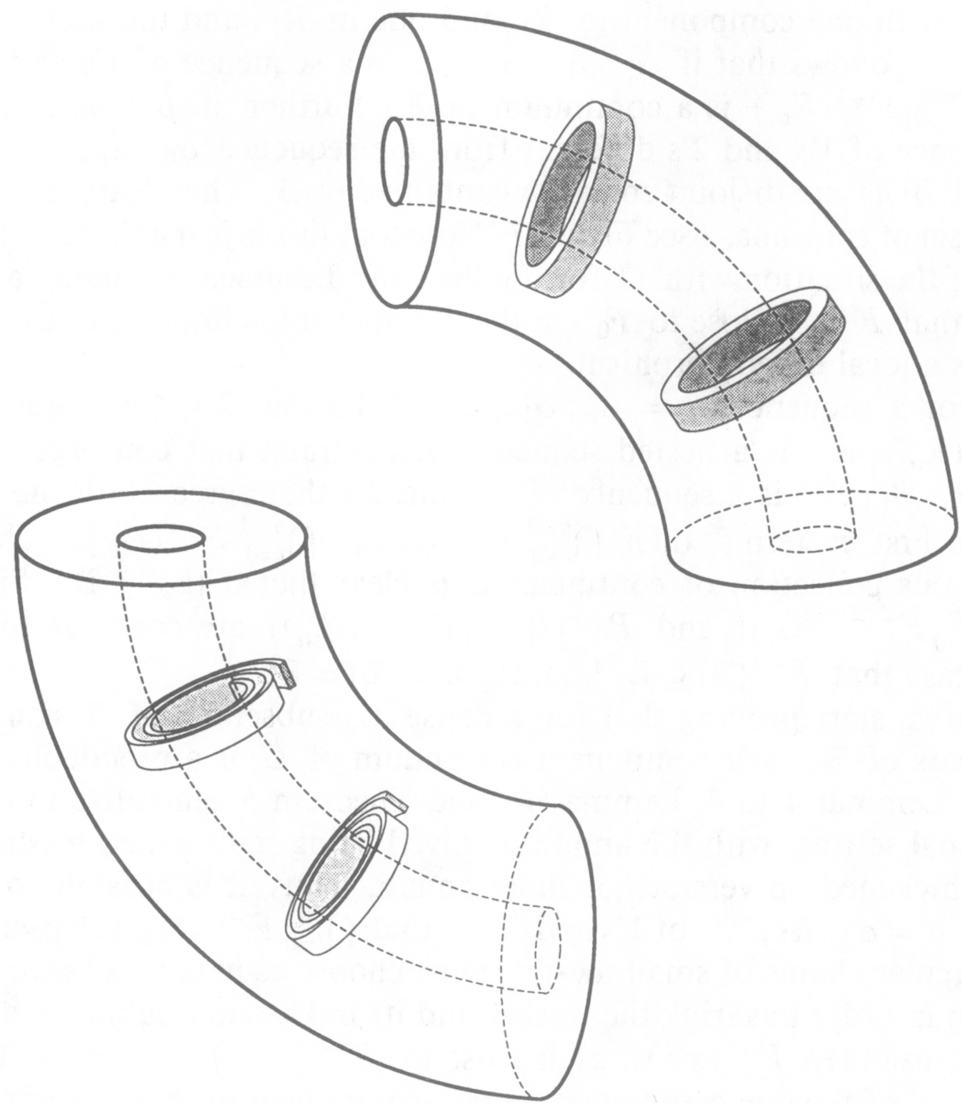

FIGURE 7

a washer as was $R_{1}$. The component of $F_{0}^{-1}\left(R_{1}\right)$ in $R_{2}$ is $\left\{\left(W(x, y), \frac{1}{3} z+\frac{2}{3}\right) \mid\right.$ $\left.(x, y, z) \in R_{1}\right\}$ and this set is just an annulus (a wiggly one; see Figure 7) crossed with an interval. Again we get a washer, although this one has wiggles. The component of $F_{0}^{-1}\left(R_{1}\right)$ in $R_{2}$ maps one-to-one onto $R_{1}$ and the component of $F_{0}^{-1}\left(R_{1}\right)$ in $R_{1}$ maps two-to-one onto $R_{1}$. Note that with respect to the lift space $\mathbb{R}^{2} \times S^{1}$, and appropriate three-dimensional strips of the form $J \times \mathbb{R}^{\times} I_{2}$, the washer $F_{0}^{-1}\left(R_{1}\right) \cap R_{2}$ has the one-component, three-component property of Lemma 4. Each slice of $F_{0}^{-1}\left(R_{1}\right) \cap R_{1}$ is a set $S^{1} \times[-1,1] \times\{z\}$ and each slice of $F_{0}^{-1}\left(R_{1}\right) \cap R_{2}$ is a set $W\left(A_{0}\right) \times\{z\}$. As for $F_{0}^{-1}\left(R_{2}\right)$, the same discussion applies (with intervals in the $z$-direction adjusted), i.e., $F_{0}^{-1}\left(R_{2}\right)$ has two components, one in $R_{1}$, which is just $S^{1} \times[-1,1] \times J$ for some interval $J$, and the other in $R_{2}$ of the form $W\left(A_{0}\right) \times J$ for some interval $J$. For $F \in N_{\varepsilon}^{1}\left(F_{0}\right)$, the situation is the same, except that now some "warping" may occur. It is still the case that for $i=1,2, F^{-1}\left(R_{i}\right) \cap R_{1}$ is a washer close to $F_{0}^{-1}\left(R_{i}\right) \cap R_{1}$ and $F^{-1}\left(R_{i}\right) \cap R_{2}$ is a wiggly washer close to $F_{0}^{-1}\left(R_{i}\right) \cap R_{2}$, but, in particular, it is no longer the case that slices in the $z$-direction go to other slices in the $z$-direction. The slices may get warped a little, but topologically the situation is the same as before.

Consider $\mathbb{B}=\left\{(x, y, z) \in S^{1} \times[-2,2] \times\left(I_{1} \cup I_{2}\right) \mid F^{n}(x, y, z) \in R_{1} \cup R_{2}\right.$ for each $n \in \widetilde{\mathbb{N}}\}$. Since $F^{-1}\left(R_{1}\right)$ has exactly two components, each of which maps 
onto $R_{1}$, with one component in $R_{1}$ and one in $R_{2}$, and the same is true for $F^{-1}\left(R_{2}\right)$, it follows that if $\alpha=\alpha_{0}, \alpha_{1}, \ldots$ is a sequence of 1's and 2's, then $B(\alpha)=\bigcap_{i=0}^{\infty} F^{-1}\left(R_{\alpha_{i}}\right)$ is a continuum in $\mathbb{B}$. Further, if $\beta=\beta_{0}, \beta_{1}, \beta_{2}, \ldots$ is a sequence of 1's and 2's different from the sequence $\alpha_{0}, \alpha_{1}, \alpha_{2}, \ldots$, then $B(\alpha)$ and $B(\beta)$ are disjoint continua contained in $\mathbb{B}$. Therefore, $\mathbb{B}$ consists of a Cantor set of continua. (See Figure 7. Note that this is just a three-dimensional version of the situation with $C$ for $F_{0}$ that was discussed two paragraphs back. The fact that $F$ is $\varepsilon$-close to $F_{0}$ (in the $C^{1}$ metric) is important, as is the fact that $F$ is a local diffeomorphism.)

Now for a sequence $\alpha=\alpha_{0}, \alpha_{1}, \ldots$ of 1's and 2's, the sequence $R_{\alpha_{0}}$, $\bigcap_{i=0}^{\infty} F^{-i}\left(R_{\alpha_{i}}\right), \ldots$ is a nested sequence of continua that converges to $B(\alpha)$. If $\beta=\beta_{0}, \beta_{1}, \ldots$ is a sequence of 1's and 2's that agrees with the sequence $\alpha$ for the first $n$ terms, then $\bigcap_{i=0}^{n-1} F^{-i}\left(R_{\alpha_{i}}\right)=\bigcap_{i=0}^{n-1} F^{-1}\left(R_{\beta_{i}}\right)$. Thus, $\mathbb{B}$ is a continuous collection of continua. It is clear that $F(\mathbb{B}) \subseteq \mathbb{B}$. Since both $R_{1} \cap\left(\bigcap_{i=0}^{\infty} F^{-i-1}\left(R_{\alpha_{i}}\right)\right)$ and $R_{2} \cap\left(\bigcap_{i=0}^{\infty} F^{-i-1}\left(R_{\alpha_{i}}\right)\right)$ are continua in $\mathbb{B}$, it is also the case that $F^{-1}(\mathbb{B}) \subseteq \mathbb{B}$, and, thus, $F(\mathbb{B})=\mathbb{B}$.

Before we start proving that for a dense $G_{\delta}$-subset $G$ of $\mathbb{B}$ consisting of components of $\mathbb{B}$, each component continuum of $G$ is a pseudocircle, please note that Lemmas 1 to 4 , Lemma $4^{\prime}$, and Theorem 5 generalize to our threedimensional setting, with the annuli involved being replaced by washers which are just thickened up versions of those annuli. Thus, it is possible to choose a sequence $\alpha=\alpha_{0}, \alpha_{1}, \ldots$ of 1's and 2's so that $\bigcap_{i=0}^{\infty} F^{-i}\left(R_{a_{i}}\right)$ is a pseudocircle. To get circular chains of small mesh we just choose as many 1's as we need at a given step in order to shrink the washer and its links, and double the number of links (because then $F^{-1}(x, y, z)$ is close to $\left.\left(T^{-1}(x, y), g^{-1}(z)\right)\right)$. To get the desired level of the one-component-three-component property, we just choose as many 2's as wee need. The 1's are used to make the resulting continuum circlelike and the 2's are used to make the small mesh circular chains "zigzag sufficiently," so that the continuum is hereditarily indecomposable in addition to being circlelike.

Suppose then that $E=e_{0}, e_{1}, \ldots, e_{n}$ is an $(n+1)$-element sequence of 1's and 2's. Let $l(E)=n$, and let $\widehat{E}=\left\{(x, y, z) \in \mathbb{B} \mid F^{i}(x, y, z) \in R_{1}\right.$ if $e_{i}=1$, and $F^{i}(x, y, z) \in R_{2}$ if $e_{i}=2$ for $\left.0 \leq i \leq l(E)\right\}$. Then $\mathscr{E}=\{\widehat{E} \mid E$ is an $(n+1)$-term sequence of 1's and 2's $\}$ for some $n \in \widetilde{\mathbb{N}}\}$ is countable with $\widehat{E}$ both open and closed in $\mathbb{B}$.

For each pair $(n, \widehat{E}) \in \mathbb{N} \times \mathscr{E}$, with $E=e_{0}, e_{1}, \ldots, e_{\hat{n}}$ there exist $m(n, \widehat{E})$ and $k(n, \widehat{E}) \in \mathbb{N}$ such that if, for each $i, 1 \leq i \leq m(n, \widehat{E}), 1=e_{\hat{n}+i}$, and for each $i, 1 \leq i \leq k(n, \widehat{E}), 2=e_{\hat{n}+m(n, \widehat{E})+i}$, then $\widehat{E}=\bigcap_{i=0}^{\hat{n}} F^{-i}\left(R_{e_{i}}\right)$, and if $\widehat{E}_{T}=\bigcap_{i=0}^{\hat{n}+m(n, \widehat{E})} F^{-i}\left(R_{e_{i}}\right)$ and $\widehat{E}_{T W}=\bigcap_{i=0}^{\hat{n}+m(n, \widehat{E})+k(n, \widehat{E})} F^{-i}\left(R_{e_{i}}\right)$, then

$$
\begin{aligned}
\mathscr{D}=\{\bar{D} \mid D \text { is a component of } & \left(\bigcap_{i=0}^{m(n, \widehat{E})+\hat{n}-1} F^{-i}\left(R_{e_{i}}\right)\right) \\
& \left.\cap F^{-\hat{n}-m(n, \widehat{E})}\left(\left(-\frac{1}{4} \cdot \frac{3}{4}\right) \times[-2,2] \times I_{1}\right)\right\}
\end{aligned}
$$


is a circular chain cover of $\widehat{E}_{T}$ of mesh less than $2^{-n}$, and (by an argument similar to that in the proof of Theorem 5) $\widehat{E}_{T W}$ "zigzags sufficiently in $\mathscr{D}$." (By "zigzags sufficiently" in $\mathscr{D}$, we mean the following: Let

$$
\widetilde{\mathscr{D}}=\left\{\overline{\widetilde{\mathscr{D}}} \mid \overline{\mathscr{D}} \text { is a component (for some } \bar{D} \in \mathscr{D} \text { ) of } \bar{D} \cap \widehat{E}_{T W}\right\} \text {. }
$$

Thus, $\widetilde{D}$ is a circular chain cover of $\widehat{E}_{T W}$. Suppose $H$ and $K$ are disjoint, closed sets of $\widehat{E}_{T W}$ with the property that if $d$ and $d^{\prime}$ are links of $\widetilde{\mathscr{D}}$ that intersect $H$ and $K$, respectively, then $d$ and $d^{\prime}$ are separated by at least 16 links. Further, suppose $U$ and $V$ are disjoint, open sets containing $H$ and $K$, respectively, such that if $d$ is a link of $\mathscr{D}$ intersecting $H$ or $K$, then the four links preceding $d$ and the four links following $d$ are all contained in $U$ or $V$. As in Theorem 5, we may then construct the sets $X_{0}, X_{1}$, and $X_{2}$ satisfying the conditions of the Krasinkiewicz-Minc theorem from the links of $\widetilde{\mathscr{D}}$.)

Let

$$
\begin{aligned}
\widetilde{E}(n)=\{( & x, y, z) \in \widehat{E} \mid F^{i}(x, y, z) \in R_{1} \text { for each } i \in \\
& \{l(E)+1, \ldots, l(E)+m(n, \widehat{E})\}, \text { and } F^{i}(x, y, z) \in R_{2} \\
& \quad \text { for each } i \in\{1+l(E)+m(n, \widehat{E}), \ldots, l(E)+m(n, \widehat{E})+k(n, \widehat{E})\}\} .
\end{aligned}
$$

Then for each $n, \widetilde{E}(n)$ is open in $\widehat{E}$, as well as closed, and $Z_{n}=\bigcup_{\dot{E} \in \mathscr{E}} \widetilde{E}(n)$ is open and dense in $\mathbb{B}$.

Further, $(x, y, z)$ is in $Z_{n}$ implies that the component of $\mathbb{B}$ that it is in has a circular chain cover $(\mathscr{D})$ of mesh less than $2^{-n}$, and the circular chain refinement $(\widetilde{D})$ for the circular chain cover $(\mathscr{D})$ "zigzags sufficiently" in the cover $(\mathscr{D})$. Then $\bigcap_{n=1}^{\infty} Z_{n}=G$ is a dense $G_{\delta}$-set in $\mathbb{B}$, and $(x, y, z) \in G$ implies the component of $\mathbb{B}$ containing $(x, y, z)$ is a pseudocircle. Thus, we have our dense $G_{\delta}$-subset of pseudocircles.

The only remaining problem is to do all this in $\mathbb{R}^{3}$, rather than in $S^{1} \times$ $\mathbb{R}^{1} \times S^{1}$. This is not difficult however. First embed $S^{1} \times[-2,2] \times I_{1}$ and $S^{1} \times[-2,2] \times I_{2}$ smoothly and disjointly in $\mathbb{R}^{3}$. (One could perhaps think of these sets in $\mathbb{R}^{3}$ as being two pieces of elbow macaroni arcing about the origin.) Then it is possible to extend $F_{0}$ defined on these copies of $S^{1} \times$ $[-2,2] \times I_{1}$, and $S^{1} \times[-2,2] \times I_{2}$ in a smooth way (using perhaps a version of the Tietze Extension Theorem), so that the resulting map on $\mathbb{R}^{3}$ has the properties desired.

\section{FUTURE GOALS}

We hope to construct improved versions of this example. We would like to construct a perturbable diffeomorphism $F$ with an extremely irregular invariant set many slices of which are generalized pseudocircles. This example will not be so easy to picture as the example constructed in this paper due to the increased number of dimensions, however.

\section{REFERENCES}

[B] R. H. Bing, Concerning hereditarily indecomposable continua, Pacific J. Math. 1 (1951).

[F1] L. Fearnley, The pseudo-circle is not homogeneous, Bull. Amer. Math. Soc. 75 (1969), 554-558. 
[F2] The pseudo-circle is unique, Bull. Amer. Math. Soc. 75 (1969), 398-401.

[Ha] Michael Handel, A pathological area preserving $C^{\infty}$ diffeomorphism of the plane, Proc. Amer. Math. Soc. 86 (1982), 163-168.

[He] Michael Herman, Construction of some curious diffeomorphisms of the Riemann sphere, $\mathbf{J}$. London Math. Soc. 34 (1986), 375-384.

[KR] J. Kennedy and J. T. Rogers, Jr., Orbits of the pseudocircle, Trans. Amer. Math. Soc. 296 (1986), 327-340.

[Kr] J. Krasinkiewicz, Mapping properties of hereditarily indecomposable continua, preprint.

[M] Richard Moeckel, Rotations of the closures of some simply connected domains, Complex Variables Theory Appl. 4 (1985), 285-294.

[OT] L. G. Oversteegen and E. D. Tymchatyn, On hereditarily indecomposable continua, Geometric and Algebraic Topology, Banach Centre Publ., vol. 18, PWN, Moscow, 1986, pp. 403413.

[PR] Ch. Pommerenke and B. Rodin, Intrinsic rotations of simply connected regions. II, Complex Variables Theory Appl. 4 (1985), 223-232.

[R] J. T. Rogers, The pseudo-circle is not homogeneous, Trans. Amer. Math. Soc. 148 (1970), 417-428.

Department of Mathematical Sciences, University of Delaware, Newark, Delaware 19716

E-mail address: jkennedy@brahms.udel.edu

Institute for Physical Science and Technology, University of Maryland, College PARK, MARYLAND 20742

E-mail address: yorke@ds2.umd.edu 